\title{
Les ceintures de l'âge du Fer en Champagne : genre et archéologie
}

Belts of the Iron Age in Champagne: gender and archeology

\section{Chloé Belard}

\section{CpenEdition \\ 1 Journals}

Édition électronique

URL : http://journals.openedition.org/clio/10804

DOI : $10.4000 /$ clio. 10804

ISSN : 1777-5299

\section{Éditeur}

Belin

\section{Édition imprimée}

Date de publication : 31 décembre 2012

Pagination : 183-190

ISSN : 1252-7017

\section{Référence électronique}

Chloé Belard, "Les ceintures de l'âge du Fer en Champagne : genre et archéologie », Clio. Femmes,

Genre, Histoire [En ligne], 36 | 2012, mis en ligne le 31 décembre 2014, consulté le 01 mai 2019. URL : http://journals.openedition.org/clio/10804; DOI : 10.4000/clio.10804 


\section{Documents}

\section{Les ceintures de l'âge du Fer en Champagne : genre et archéologie}

Chloé BELARD

En archéologie protohistorique européenne, les costumes entièrement et véritablement préservés sont exceptionnels et nécessitent des conditions climatiques de conservation particulières. Le plus souvent, il ne subsiste que les accessoires vestimentaires métalliques, comme les fibules ou certains éléments de ceinture.

L'étude, présentée ici, porte sur les ceintures des ensembles sépulcraux de Champagne à l'âge du Fer. Cette période couvre la quasi-totalité du premier millénaire avant notre ère et plus précisément de l'extrême fin du IX siècle à la seconde moitié du I $^{\text {er }}$ siècle av. J.-C. La phase retenue s'étend du dernier tiers du VI ${ }^{\mathrm{e}}$ siècle au $\mathrm{III}^{\mathrm{e}}$ siècle av. J.-C. inclus.

Le choix de la Champagne a été motivé par le fait, tout d'abord, qu'elle a livré plusieurs milliers de tombes, fouillées (pour la plus grande part) avant la Première Guerre mondiale ${ }^{1}$. Les données archéologiques exploitables représentent donc une quantité d'informations importante, bien que leur qualité soit bien souvent très inégale. Par ailleurs, la chronologie, construite à partir des évolutions typologiques des objets, est bien établie. La typo-chronologie - à la base de toute étude archéologique - étant constituée, nous pouvons

1 Demoule 1999. 
tenter d'aller plus loin dans la compréhension de la construction des identités de genre perceptibles dans les ensembles funéraires.

Il s'agit de comprendre de quelle manière la ceinture - appréhendable seulement s'il y a eu conservation d'éléments métalliques participe à la constitution des différences entre les genres des défunts, matérialisés dans leur ultime costume.

Au premier abord, la question des ceintures à l'âge du Fer en Champagne apparaitt extrêmement claire et définie. Étant donné leur très petit nombre dans les tombes d'enfants, il y aurait des ceintures "d'hommes », qui auraient une fonction bien précise - celle de suspendre l'épée ou le poignard au niveau de la taille - et des ceintures «de femmes", dont la fonction serait principalement vestimentaire et ornementale.

Les types de ceintures qui sont, la plupart du temps, retenus dans les études, appartiennent généralement aux phases chronologiques de la fin du IVe et du III ${ }^{e}$ siècle av. J.-C. Ils sont entièrement métalliques (cf. l'illustration), contrairement à ceux des périodes précédentes, constitués d'une agrafe et/ou anneaux en métal (alliage cuivreux ou fer), les liens entre ces éléments étant en matériaux périssables. Ces types récents sont les plus étudiés, du fait de leur facture extrêmement soignée et de leur "valeur chronologique», car ce sont des types caractéristiques, bien datés à partir de leur contexte sépulcral.

Or, en prenant en compte tous les éléments de ceinture sur l'ensemble de la période concernée, il apparaît que les résultats sont bien moins clivés que ce que l'on a avancé au départ. La détermination sexuelle du défunt par l'anthropologie biologique apporte des informations qui nuancent cette dualité.

Tout d'abord, deux femmes, dont le sexe a été déterminé anthropologiquement, ont été inhumées avec une ceinture servant à suspendre un couteau à leur taille. Plusieurs hommes portaient des éléments de ceinture métalliques, sans que ceux-ci soient associés avec une arme de poing. Ces exemples, quoiqu'encore peu nombreux, permettent d'atténuer les fonctions que l'on assigne traditionnellement aux ceintures trouvées en contextes dits « masculin » ou « féminin ». 


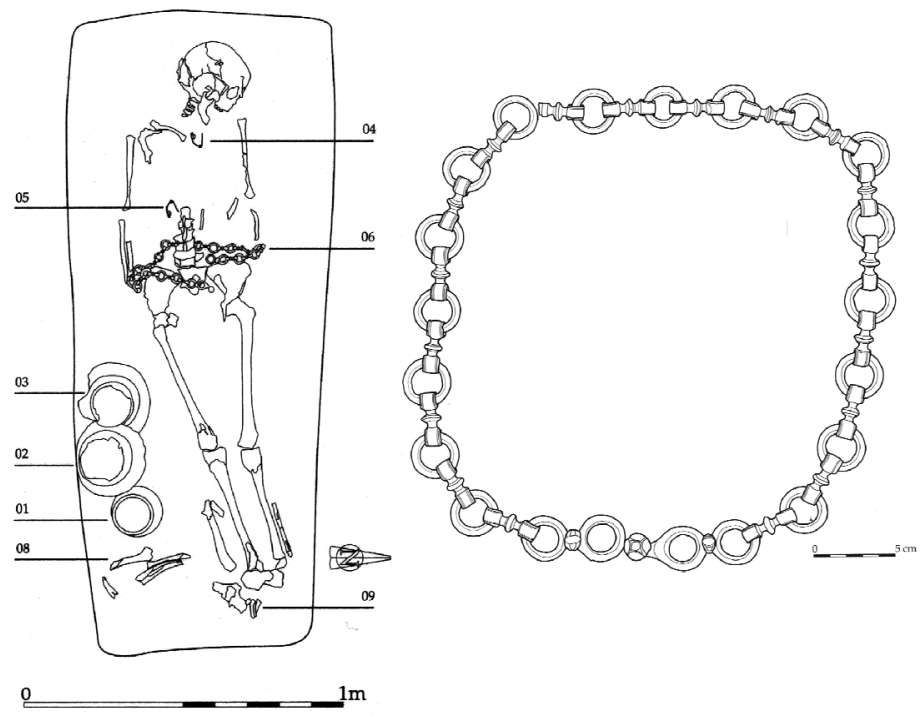

Sépulture 4 de la nécropole d'Orainville "La Croyère" (Aisne), $n^{\circ} 4$ et 5 : fibules ; $n^{\circ} 6$ : ceinture (d'après Desenne et al. 2005 , fig. 42 et 43 ).

Mais les résultats les plus étonnants concernent les types de ceintures en eux-mêmes. En effet, près de la moitié des ceintures appartiennent à des types communs à tous les défunts enterrés avec des éléments de ceinture. Ce sont des objets peu caractéristiques, comportant un à cinq anneaux, en fer ou en alliage cuivreux et associés ou non à une agrafe de ceinture. Leur contexte sépulcral permet de les dater généralement de la fin $\mathrm{du} \mathrm{VI}^{\mathrm{e}}$ à la première moitié du IVe siècle av. J.-C.

Les ceintures de cette période ne participent donc pas à la construction sociale des différences entre les hommes et les femmes dans les nécropoles. Elles ne semblent pas être investies d'une signification particulière, tout du moins en ce qui concerne leur typologie et leur facture, car leur position dans la tombe peut être singulière et offre un autre niveau d'analyse. 
Dès la fin du IVe siècle av. J.-C., il semble que les ceintures acquièrent une importance symbolique significative, qui peut être perçue à travers deux indications singulières.

Tout d'abord, le nombre de défunts inhumés avec des éléments de ceinture métalliques augmente significativement, passant de 6-16\% pour les phases chronologiques précédentes à $29 \%$ de la totalité des individus, soit un peu moins d'un défunt sur trois. Cette proportion est donc tout à fait conséquente et témoigne d'une volonté, non pas d'accroître véritablement le nombre de dépôts de ceintures en contexte sépulcral - des ceintures en matières périssables pouvaient être placées dans les tombes des périodes précédentes, mais d'investir et de pérenniser techniquement et symboliquement ces ceintures.

De plus, les ceintures évoluent vers des types où tous les éléments sont en métal, que ce soient les agrafes, les anneaux, mais aussi les pièces reliant ces éléments. Ce fait est directement corrélé avec le précédent. La masse de métal dévolue à la fabrication de ces ceintures augmente significativement, ainsi que le soin porté à leur facture, ce qui traduit, là encore, la nouvelle importance symbolique conférée à cette époque aux éléments de ceinture.

Les ceintures acquièrent donc, à partir de la fin du IVe siècle av. J.-C., un statut tout à fait unique pour le second âge du Fer (seconde moitié $\mathrm{du} \mathrm{V}^{\mathrm{e}}$ - deuxième moitié du Ier siècle av. J.-C.). Cette évolution et le nouveau statut qui semble leur avoir été conféré sont communs à toutes les ceintures déposées dans les ensembles funéraires de Champagne.

C'est seulement à partir de cette période que des types de ceintures sont réservés soit aux tombes d'hommes - uniquement à armes de poing -, soit aux tombes de femmes - contenant ou non des éléments de parure -, signifiant un changement de l'usage de la ceinture 2 .

En effet, pour les tombes d'hommes, seules celles renfermant des épées contiennent aussi des éléments de ceinture, car ces derniers, appelés "chaines de suspension ", sont à présent fabriqués avec des maillons articulés, permettant une suspension souple et stable de l'épée à la taille et une bonne adaptation aux mouvements du corps ${ }^{3}$. La ceinture fait donc ici davantage partie de l'armement que des

Baray 2008.

Rapin 1991 par exemple. 
accessoires vestimentaires, comme nous l'indique aussi sa position dans la tombe, qui n'est plus à la taille du défunt, mais qui est située désormais sur l'épée et le fourreau, déposés le plus souvent le long d'un des deux bras du mort. La position n'est donc pas «fonctionnelle », comme nous le verrons plus loin.

Dans les tombes de femmes, cette dissociation entre la fonction et l'usage de la ceinture n'est pas aussi claire. Mais la facture nettement imposante et ostentatoire des ceintures (cf. l'illustration) permet toutefois de suggérer un changement de signification symbolique de ces objets, qui peut être mis aussi en relation avec la "désaffection » du torque - collier rigide en métal, indiquant probablement un statut social particulier pour le défunt qui le porte - à cette même période. Cet élément de parure caractéristique est effectivement présent dans les tombes à partir du dernier tiers du VIe siècle av. J.-C. en Champagne, mais tend à se raréfier et à être de facture moins soignée à partir du début du $\mathrm{III}^{\mathrm{e}}$ siècle av. J.-C., pour disparaittre totalement des ensembles sépulcraux à la fin du $\mathrm{III}^{\mathrm{e}}$-début $\mathrm{II}^{\mathrm{e}}$ siècle av. J.-C.

Les ceintures deviennent, à partir de la fin du IV siècle av. J.-C., des objets nous renseignant sur le genre «sexuellement connoté $»^{4}$ du défunt qui est inhumé avec, mais elles ne peuvent pas être considérées comme un critère exclusif et discriminant, encore moins comme un «marqueur sexuel $»^{5}$, car elles ne se trouvent pas dans la totalité des tombes des nécropoles de cette période. Elles entrent donc dans les catégories d'objets qui peuvent nous informer sur le statut social du défunt, dont l'expression matérielle peut être corrélée avec son genre.

Toutefois, leur localisation dans la tombe n'est pas nécessairement liée à leur fonction première d'accessoire vestimentaire. Ces variations dans leur position sont à prendre en compte pour l'attribution de ces objets aux biens possédés par le défunt de son vivant.

Ces ceintures appartiennent-elles réellement aux défunts?

La ceinture peut être déposée, dans une sépulture, de trois manières différentes. La première est la position dite strictement «fonctionnelle », c'est-à-dire que la ceinture est véritablement portée par le

\footnotetext{
Héritier $2010: 29$.

5 Expression généralement attribuée, en archéologie, aux objets de la panoplie « personnelle » du défunt, comme les armes ou certains éléments de parure.
} 
défunt lors de son inhumation (comme sur l'illustration), ce qui est assez difficile à mettre en évidence avec certitude lorsque la ceinture n'est pas entièrement métallique. Dans ce cas, la ceinture est considérée comme appartenant au défunt qui la porte. Il faudrait néanmoins nuancer cette idée, notamment lorsque la ceinture présente des traces d'usure importante alors que le défunt a un âge au décès peu élevé.

Le deuxième cas concerne les ceintures qui sont simplement déposées sur le bassin du défunt. C'est le plus courant, bien que certains dépôts soient peut-être plus à placer dans le premier cas. Mais par manque d'informations plus précises, on ne peut bien souvent pas statuer. Dans ces sépultures, le défunt ne porte donc pas réellement la ceinture, malgré la volonté de conserver l'emplacement «fonctionnel ». Pour les vivants qui ont procédé à la déposition, le défunt doit uniquement emporter la ceinture avec lui dans la tombe.

Enfin, le troisième cas regroupe les éléments de ceinture qui sont déposées hors du volume initial du corps. Les localisations peuvent être variées : au niveau du crâne du défunt, de ses bras ou de ses jambes. Ce simple dépôt de ceintures dans la sépulture nous invite à nuancer leur appartenance au défunt.

Cela nous permet de modérer notre approche interprétative, qui considère que tous les objets, en dehors des offrandes céramiques et alimentaires, appartiennent à la panoplie personnelle du défunt. On ne peut pas, à partir des différences dans la composition des ensembles mobiliers, établir de manière directe et incontestable les identités personnelle et sociale du défunt. La constitution des dépôts mobiliers dans les tombes est avant tout tributaire du choix et des actes des vivants, qui, bien qu'ils soient soumis aux normes sociales et à la volonté du défunt, cherchent aussi à modifier, renégocier et légitimer les liens sociaux qui les unissent au mort, mais également entre eux ${ }^{6}$. Cette construction des assemblages des objets des ensembles sépulcraux nous donne donc accès à la catégorie de genre attribuée au défunt, définie par le contexte social, et non à son identité de genre (définie par l'individu lui-même) ou, encore moins, à son sexe biologique?

\footnotetext{
Lemonnier $2008: 210-211$

Arnold 2001 : 242.
} 
L'existence de types de ceintures nous renseignant sur le genre du défunt n'intervient qu'à partir de la fin du IVe siècle av. J.-C., période à laquelle la ceinture acquiert un statut inédit et singulier pour le second âge du Fer, tant au niveau du nombre de défunts qui sont inhumés avec, que de la masse de métal investie et du soin apporté à sa facture. Elle est investie d'un intérêt particulier et revêt une nouvelle symbolique. La ceinture devient donc un objet participant avant tout à la signification du statut social privilégié du défunt, dont la typologie peut être corrélée avec son appartenance à une catégorie de genre. Malgré une fonction clairement définie, il apparait donc que l'usage funéraire de la ceinture est bien plus complexe qu'on pouvait le penser à priori.

\section{Bibliographie}

ARNOLD Bettina, 2001, "Sein und Werden: Gender as process in mortuary ritual», in Sarah M. NELSON (ed.), In the pursuit of gender, Walnut Creek, AltaMira, p. 239-256.

ARNold Bettina \& Nancy L. WiCKer (eds), 2001, Gender and the archaeology of death, Walnut Creek, AltaMira.

BARAy Luc, 2003, Pratiques funéraires et sociétés de l'âge du Fer dans le Bassin parisien : fin du VII s.-troisième quart du II s. avant J.-C., Paris, CNRS Éditions.

—, 2008, «Dimension socio-économique et symbolique des dépôts funéraires aristocratiques d'Europe occidentale (VIII ${ }^{\mathrm{e}} \mathrm{I}^{\mathrm{er}} \mathrm{s}$ s. av. J.-C.) ", in Maxence BAILLY \& Hugues PLISSON (dir.), La valeur fonctionnelle des objets sépulcraux, Aix-en-Provence, APPAM, p. 183-208.

Demoule Jean-Paul, 1999, Chronologie et société dans les nécropoles celtiques de la culture Aisne-Marne du VI au III ${ }^{e}$ s. avant notre ère, Amiens, Service Régional de l'Archéologie en Picardie.

Desenne Sophie, Collart Jean-Luc, Auxiette Ginette et al., 2005, «La nécropole d'Orainville "La Croyère" (Aisne). Un ensemble attribuable au Aisne-Marne IV », Revue Archéologique de Picardie, numéro spécial 22, p. 233-287.

GILCHRIST Roberta, 1999, Gender and archaeology: contesting the past, London, New York, Routledge.

HérITIER Françoise, 2010, Hommes, femmes : la construction de la différence, Paris, Le Pommier. 
Lemonnier Pierre, 2008, «La mort, le mort, les morts et les autres. Remarques d'anthropologue», in Maxence BAILLY \& Hugues PLISSON (dir.), La valeur fonctionnelle des objets sépulcraux, Aix-en-Provence, APPAM, p. 209-214.

NeLSON Sarah M. (ed.), 2007, Women in Antiquity: Theoretical approaches to gender and archaeology, Walnut Creek, AltaMira.

RAPIN André, 1991, «Le ceinturon métallique et l'évolution de la panoplie celtique au III siècle av. J.-C. », Études celtiques, 28, p. 349-368.

Sørensen Marie Louise Stig, 2000, Gender archaeology, Cambridge, Polity Press. 